\title{
Internalizing symptoms associate with the pace of epigenetic aging in childhood
}

\author{
Marieke S. Tollenaar $^{\mathrm{a}, *}$, Roseriet Beijers ${ }^{\mathrm{b}, \mathrm{c}}$, Elika Garg ${ }^{\mathrm{d}}$, T.T. Thao Nguyen ${ }^{\mathrm{d}}$, David T.S. Lin ${ }^{\mathrm{e}}$, \\ Julia L. MacIsaac ${ }^{e}$, Idan Shalev ${ }^{f}$, Michael S. Kobor ${ }^{e, g}$, Michael J. Meaney ${ }^{\text {d, g,h,i, }}$ \\ Kieran J. O'Donnell ${ }^{\mathrm{d}, \mathrm{h}, \mathrm{j}, 1}$, Carolina de Weerth ${ }^{\mathrm{c}, 1}$ \\ ${ }^{a}$ Institute of Psychology, Leiden University, the Netherlands \\ ${ }^{\mathrm{b}}$ Department of Developmental Psychology, Behavioural Science Institute, Radboud University, the Netherlands \\ ${ }^{\mathrm{c}}$ Department of Cognitive Neuroscience, Donders Institute for Brain, Cognition and Behaviour, Radboud University Medical Center, the Netherlands \\ ${ }^{\mathrm{d}}$ Ludmer Centre for Neuroinformatics and Mental Health, Douglas Hospital Research Centre, McGill University, QC, Canada \\ ${ }^{\text {e }}$ Centre for Molecular Medicine and Therapeutics, Department of Medical Genetics, BC Children's Hospital Research Institute, University of British Columbia, BC, \\ Canada \\ ${ }^{\mathrm{f}}$ Department of Biobehavioral Health, Pennsylvania State University, PA, USA \\ ${ }^{\mathrm{g}}$ Sackler Program for Epigenetics and Neurobiology, McGill University, QC, Canada \\ ${ }^{\mathrm{h}}$ Canadian Institute for Advanced Research, Child and Brain Development Program, Canada \\ ${ }^{\mathrm{i}}$ Singapore Institute for Clinical Sciences, Singapore \\ ${ }^{\mathrm{j}}$ Yale Child Study Center \& Department of Obstetrics, Gynecology and Reproductive Sciences, Yale School of Medicine, USA
}

\section{A R T I C L E I N F O}

\section{Keywords:}

Childhood

Internalizing symptoms

Externalizing symptoms

Epigenetic age acceleration

Biological aging

\begin{abstract}
A B S T R A C T
Childhood psychiatric symptoms may be associated with advanced biological aging. This study examined whether epigenetic age acceleration (EAA) associates with internalizing and externalizing symptoms that were prospectively collected across childhood in a longitudinal cohort study. At age 6 buccal epithelial cells from 148 children (69 girls) were collected to survey genome-wide DNA methylation. EAA was estimated using the Horvath clock. Internalizing symptoms at ages 2.5 and 4 years significantly predicted higher EAA at age 6 , which in turn was significantly associated with internalizing symptoms at ages 6-10 years. Similar trends for externalizing symptoms did not reach statistical significance. These findings indicate advanced biological aging in relation to child mental health and may help better identify those at risk for lasting impairments associated with internalizing disorders.
\end{abstract}

\section{Introduction}

Common mental health disorders, such as anxiety and depression, result in chronic human suffering and come at a large economic cost (Whiteford et al., 2013). As psychiatric symptoms often have their origins early in life (Reef, Diamantopoulou, van Meurs, Verhulst, \& van der Ende, 2009), early indicators of childhood psychiatric symptoms are of high importance. Recent studies show that depressive symptoms in childhood and adolescence may be associated with advanced physical aging, such as early pubertal and adrenarcheal timing (Copeland, Worthman, Shanahan, Costello, \& Angold, 2019; Ellis et al., 2019; Lewis et al., 2018), possibly due to early life adversity (Gur et al., 2019). Here we examine whether an indicator of advanced biological aging based on genomic DNA methylation, i.e., epigenetic age acceleration (EAA), is associated with childhood psychiatric symptoms.

Epigenetic age has recently emerged as a cross-tissue index of biological aging (Horvath \& Raj, 2018). EAA has been associated with cancer, cardiovascular disesase and all-cause mortality, independent of chronological age (Chen et al., 2016; Marioni et al., 2015; Perna et al., 2016), although not in all studies (Kim et al., 2018; Murabito et al., 2018). EAA is furthermore predicted by chronic life stress (Gassen, Chrousos, Binder, \& Zannas, 2017; Zannas et al., 2015), and has been associated with mental health problems in adult populations (Fries et al., 2017; Han et al., 2018; Wolf \& Schnurr, 2016).

\footnotetext{
* Corresponding author at: Institute of Psychology, Leiden University, and Leiden Institute for Brain and Cognition, Wassenaarseweg 52, PO box 9555, 2300 RB, Leiden, the Netherlands.

E-mail address: m.s.tollenaar@fsw.leidenuniv.nl (M.S. Tollenaar).

${ }^{1}$ Denotes joint senior-author status.
} 
Previous studies indicate that EAA is also an indicator of advanced physical maturation in younger populations, as demonstrated by early pubertal development and higher cortisol output in adolescence (Binder et al., 2018; Davis et al., 2017; Suarez, Lahti, Czamara, Lahti-Pulkkinen, Girchenko et al., 2018). A longitudinal study in a pediatric sample from birth to age 17 also suggests that EAA is associated with advanced maturation in certain physical domains, e.g. fat mass and height, although not in others (e.g. not with puberty) (Simpkin et al., 2017). Importantly, EAA is also associated with higher odds for psychiatric problems in adolescent girls (Suarez, Lahti, Czamara, Lahti-Pulkkinen, Girchenko et al., 2018). However, associations between EAA and psychiatric symptoms in younger children remain unknown.

We generated EAA estimates from buccal cells at age 6 in a longitudinal study sample, and examined whether it associates with trajectories of internalizing and externalizing symptoms across childhood. We first examined whether early childhood symptoms predicted EAA at age 6 , and secondly whether EAA at age 6 would predict symptom development at ages 6-10.

\section{Method}

\subsection{Participants}

Participants are children of an ongoing longitudinal study including 193 mothers and children (Beijers, Jansen, Riksen-Walraven, \& de Weerth, 2010; Tollenaar, Beijers, Jansen, Riksen-Walraven, \& De Weerth, 2011). At age 6 years, genomic DNA was extracted from buccal epithelial cells in 148 of these children (69 girls, 79 boys). Mothers gave informed consent, and the local Institutional Ethics Committee, which follows the Helsinki Declaration, approved the study protocols. See the Supplementary information for further details on the study sample.

\subsection{Psychiatric symptoms}

Maternal reports were used to index childhood internalizing and externalizing symptoms prospectively over time. At ages 2.5 and 4 years the CBCL 1.5-5 (Achenbach \& Rescorla, 2000) was used and at ages 6, 7 and 10 the CBCL 4-18 (Achenbach, 2007). Internalizing and externalizing symptoms at ages 8 and 10 were also measured with the SDQ (Mieloo et al., 2014), to confirm if study outcomes are consistent across symptom checklists. Continuous symptom scale scores were used in the analyses, but CBCL t-scores are included in Table 1 for reference.

We also considered maternal anxiety and depressive symptoms at ages 2.5, 4, 6, 8 and 10 to ensure our results were not confounded by maternal mood at time of reporting child symptoms. Maternal symptoms of anxiety and depression were assessed using the State-Trait Anxiety Inventory (STAI) (Spielberger, 1983) and the Edinburgh Postnatal Depression Scale (EPDS) (Pop, Komproe, \& van Son, 1992) respectively.

\subsection{Epigenetic Age Acceleration (EAA) and genetic covariates}

Genome-wide DNA methylation was assessed with the Infinium EPIC (850 K) array and genotyping was performed using the Infinium Global Screening Array (see Supplementary information for details). Epigenetic age was calculated with the script developed by Horvath (2013), as this clock is known as pan tissue epigenetic clock and has shown associations with development in childhood before (Binder et al., 2018; Davis et al., 2017; Simpkin et al., 2017; Suarez, Lahti, Czamara, Lahti-Pulkkinen, Girchenko et al., 2018). EAA was estimated by the residuals that result from regressing epigenetic age on chronological age. A positive EAA indicates higher than expected epigenetic age, whereas a negative EAA indicates lower than expected epigenetic age.

To control for cell heterogeneity (Zheng et al., 2018), we used an approach informed by Smith et al. (2015) to estimate buccal epithelial cell content and blood cell contamination (CD34+ cells). Since these showed a very high correlation $(r=-0.93, p<.001)$ only buccal epithelial cell content was included as a covariate. We used a well-established Principal Component (PC) analysis-based approach to control for population stratification (Price et al., 2006). From this analysis the first 2 PCs were identified to best describe the population structure of our cohort and were included as covariates. We also considered potential genetic risk factors for EAA based on studies in brain and blood (2018, Lu et al., 2016). However, none of these genetic risk markers contributed to EAA (see Supplementary information) and were hence not included in further analyses.

\subsection{Statistical analyses}

IBM SPSS 24 was used for all analyses with $\alpha$ at .05 . Analyses that were separately performed for internalizing and externalizing symptoms had $\alpha$ corrected to .025. To account for the non-normal distributed CBCL and SDQ data, Spearman rank correlations were used in the descriptive analyses, and log-transformed values in the main analyses.

For the prediction of EAA the following covariates were examined: child sex, buccal cell content, $850 \mathrm{~K}$ array technical factors (batch number, array plate, array position), and population stratification (PC1 and PC2). For the prediction of CBCL scores over time covariates included: child sex, maternal educational level at birth, mothers' own anxiety and depression ratings per time point, and population stratification (PC1 and PC2), as well as any significant covariates of EAA. All covariates were at once included in a first step in the regression and mixed models. Only significant covariates $(p<.05)$ were kept in the main analyses to increase power by reducing the number of predictors in relation to the sample size.

Linear regression analyses were performed with internalizing and externalizing symptoms at ages 2.5 and 4 as predictors of EAA at age 6 . Covariates were examined as described above. To examine whether increases in symptoms at age 4 compared to age 2.5 would associate

Table 1

Descriptive statistics for internalizing and externalizing symptoms from ages 2.5 to 10 years.

\begin{tabular}{|c|c|c|c|c|c|c|c|}
\hline & $\begin{array}{l}\text { CBCL }^{\mathrm{a}} \\
\text { Age } 2.5\end{array}$ & $\begin{array}{l}\text { CBCL }^{a} \\
\text { Age } 4\end{array}$ & $\begin{array}{l}\mathrm{CBCL}^{\mathrm{b}} \\
\text { Age } 6\end{array}$ & $\begin{array}{l}\mathrm{CBCL}^{\mathrm{b}} \\
\text { Age } 7\end{array}$ & $\mathrm{CBCL}^{\mathrm{b}}$ Age 10 & $\begin{array}{l}\text { SDQ } \\
\text { Age } 8\end{array}$ & $\begin{array}{l}\text { SDQ } \\
\text { Age } 10\end{array}$ \\
\hline $\mathrm{N}$ & 145 & 139 & 143 & 143 & 133 & 145 & \\
\hline \multicolumn{8}{|l|}{ Internalizing Symptoms } \\
\hline Mean & 7.20 & 6.33 & 4.09 & 4.61 & 5.60 & 3.21 & 2.80 \\
\hline SD & 4.78 & 4.96 & 3.95 & 4.44 & 5.47 & 2.80 & 2.66 \\
\hline Range & $0-21$ & $0-24$ & $0-21$ & $0-24$ & $0-35$ & $0-14$ & $0-15$ \\
\hline Mean t-score(range) & $48.0(29-66)$ & $46.0(29-69)$ & $48.3(32-73)$ & $49.4(32-75)$ & $50.3(33-80)$ & & \\
\hline \multicolumn{8}{|l|}{ Externalizing Symptoms } \\
\hline Mean & 9.68 & 11.05 & 6.55 & 6.43 & 6.31 & 4.83 & 4.02 \\
\hline SD & 4.88 & 7.07 & 5.23 & 5.73 & 5.96 & 3.47 & 3.29 \\
\hline Range & $0-21$ & $0-33$ & $0-26$ & $0-29$ & $0-30$ & $0-17$ & $0-16$ \\
\hline Mean t-score(range) & $50.8(28-71)$ & $47.7(28-73)$ & $49.3(32-72)$ & $48.6(32-74)$ & $50.5(33-74)$ & & \\
\hline
\end{tabular}

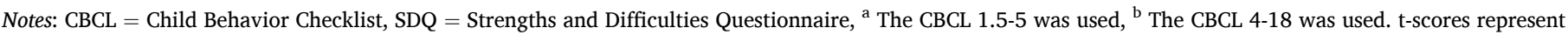
normative gender-adjusted values of the CBCL for the respective age groups. 
with higher levels of EAA, scores at 2.5 and 4 were included together in a final regression model. Increases in $R^{2}\left(\Delta R^{2}\right)$ were used as indicators of model fit and reflected additionally variance explained per model.

Linear mixed model analyses were performed with EAA at age 6 as a predictor of internalizing and externalizing symptoms at ages 6,7 , and 10 assessed with the CBCL. Mixed models allow for the inclusion of time varying components, and for individual missing values between time points. The intercept was included as a random factor. In addition to the covariates, linear and curvilinear time components were examined in the first step to assess development of symptoms from age 6-10. Then EAA was added as a main effect, and subsequently in interaction with time to examine whether EAA would also predict an increase in symptom development over time. Lastly, internalizing and externalizing symptoms at ages 2.5 and 4 were included to examine whether EAA could predict symptoms at ages $6-10$ beyond early childhood symptoms alone. Changes in random intercept variance were used as an estimate of additionally explained variance between individuals. As confirmatory analyses, similar mixed model analyses were performed for internalizing and externalizing symptoms assessed with the SDQ at ages 8 and 10 as outcome measure.

\section{Results}

The average chronological age of the participants at the time of DNA sampling was 6.09 years ( $S D=0.14$, range: $5.87-6.85$ years). The average epigenetic age was 4.64 years $(S D=.80$, range: $3.07-6.93$ years), which was significantly lower than chronological age, $t(147)=$ $21.8, p<.001$, and not correlated to it $(r=.077, p=.36)$. Measures of EAA ranged from -1.58 to 2.21 years, with an average of $0(S D=.80)$, and correlated very strongly with epigenetic age $(r=.997, p<.001)$. Boys and girls did not differ in EAA, $t(146)=-.30, p=.77$.

Table 1 shows internalizing and externalizing symptoms from ages 2.5 to 10 . One participant, who was diagnosed with an autism spectrum disorder by age 10, had high internalizing and externalizing symptoms ( $>3$ SD from the mean) on 3 occasions and was removed from subsequent analyses as a repeated outlier. No sex differences were found on the CBCL scales at any age (all ps $>.07$ ). At age 10 only, girls exhibited higher levels of SDQ-reported internalizing problems than boys $(F[1$, $135]=4.03, p=.04)$. Symptom scores over time were positively correlated (see Table 2), and internalizing and externalizing symptoms were positively correlated at all time points (see Supplementary Table S1). Table 2 also shows positive correlations between EAA at age 6 and psychiatric symptoms over time, indicating both cross-sectional and longitudinal positive associations, mainly for internalizing symptoms. Bivariate associations between internalizing and externalizing symptoms scores over time, EAA and all covariates can be found in Supplementary Tables S2 and S3.

\subsection{Predicting EAA at age 6 from childhood psychiatric symptoms}

Linear regression analyses were performed to predict EAA at age 6 . Covariates were first examined as predictors of EAA. Array position was the only significant covariate $(b=-.30, p=.006)$ and subsequently included in the regression models. Internalizing symptoms at age 2.5 significantly predicted EAA $\left(b=.20, p=.015, \Delta R^{2}=.04\right)$. Internalizing symptoms at age 4 also significantly predicted EAA $(b=.19, p=.024$, $\Delta R^{2}=.035$ ). When symptoms at age 4 were added to model with symptoms at age 2.5 , no significant additional variance was explained $\left(\Delta R^{2}=.003, p=.53\right)$. See Supplementary Table S4 for an overview of the regression models. To visualize the association between early childhood internalizing symptoms and EAA, Fig. 1.A shows a scatterplot of the association between standardized (log-transformed) internalizing symptoms at age 2.5 and EAA at age 6 .

Externalizing symptoms at age 2.5 did not significantly predict EAA ( $b=.12, p=.14, \Delta R^{2}=.015$ ), and neither did externalizing symptoms at age $4\left(b=.12, p=.16, \Delta R^{2}=.015\right)$. When both externalizing symptoms at ages 2.5 and 4 were included, EAA was not significantly predicted by either ( $p s>.05$, see Supplementary Table S5). Furthermore, when externalizing symptoms at age 2.5 were added to a model with internalizing symptoms at age 2.5 , this did not account for additional variance in EAA $\left(\Delta R^{2}<.001, p=.83\right.$; externalizing symptoms: $b$ $=.021, p=.83$, internalizing symptoms: $b=.18, p=.065$ ).

\subsection{Predicting childhood psychiatric symptoms over time from EAA at age 6}

\subsubsection{Internalizing symptoms}

Covariate analyses showed that time as a linear factor (beta $=.048$, $\mathrm{SE}=.014, t=3.54, p<.001$ ), PC1 (beta $=-.901, \mathrm{SE}=.346, t=-2.60, p$ $=.01$ ) and maternal STAI scores (beta $=.0096, \mathrm{SE}=.0026, t=3.66, p<$

Table 2

Spearman rank correlations between CBCL and SDQ scales over time and EAA, for internalizing and externalizing symptoms separately.

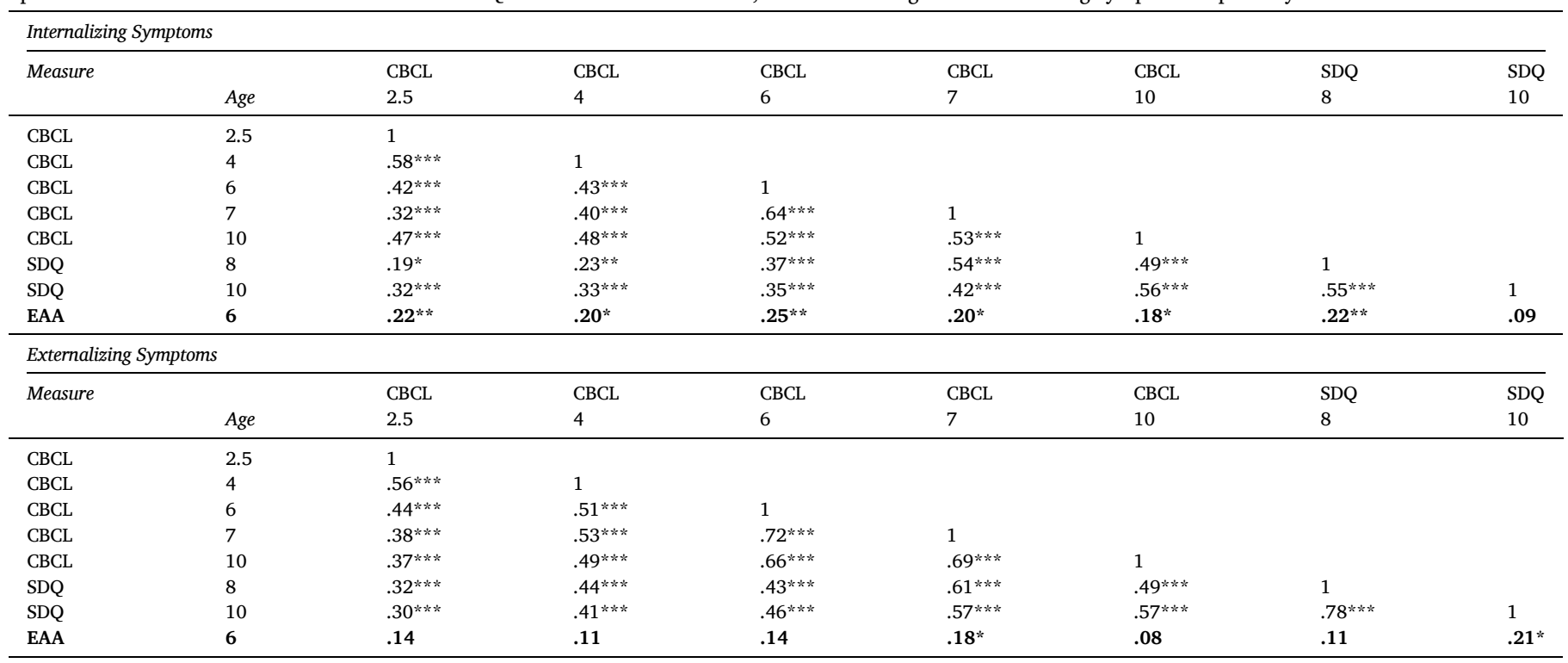

Notes: $\mathrm{CBCL}=$ Child Behavior Checklist, $\mathrm{SDQ}=$ Strengths and Difficulties Questionnaire, EAA $=$ Epigenetic Age Acceleration, Age in years, ${ }^{*} \mathrm{p}<.05,{ }^{* *} \mathrm{p}<.01, * * * \mathrm{p}$ $<.001$. 
A.

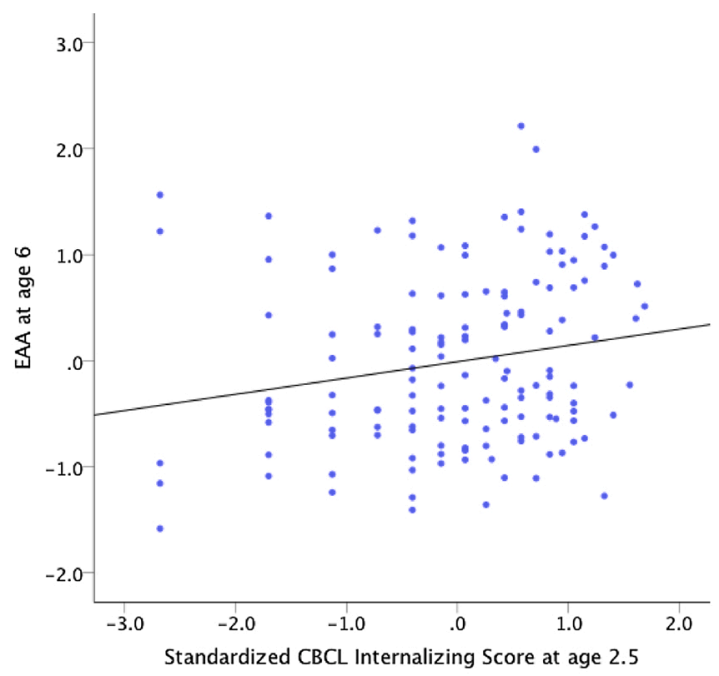

B.

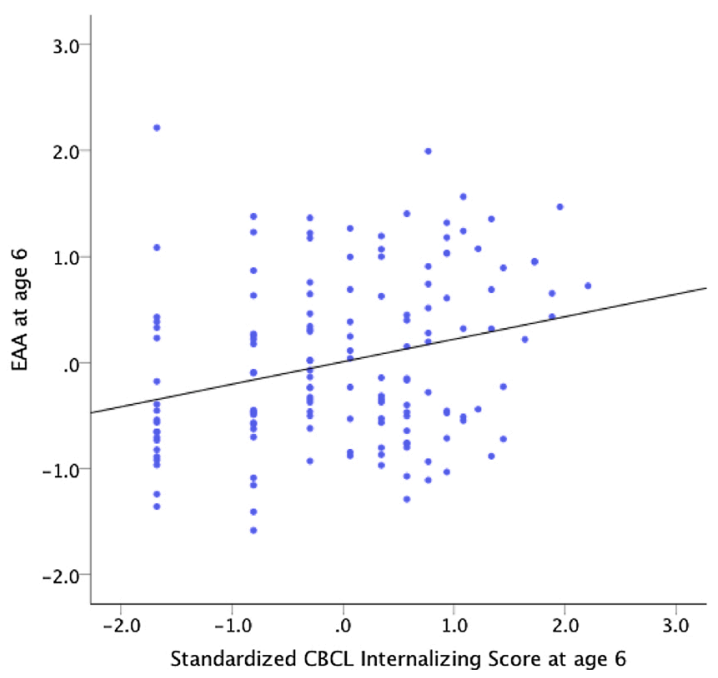

Fig. 1. Associations between standardized internalizing scores at age 2.5 (1.A) and 6 (1.B) measured with the Child Behavior Checklist (CBCL) and Epigenetic Age Acceleration (EAA) at age 6 .

.001) significantly predicted CBCL internalizing symptoms at ages 6-10, indicating an increase in internalizing symptoms over time, and positive associations between maternal anxiety problems and her report on child internalizing symptoms.

EAA was associated with significantly higher levels of internalizing symptoms at ages $6-10$ (beta $=.096, \mathrm{SE}=.028, t=3.42, p=.001$ ), explaining $9.4 \%$ of variance between individuals. The interaction of EAA with time was not significant $(p>.65$, see Supplementary Table S6 for an overview of these mixed model analyses). Including array position as a potential confounder did not affect the results. We then examined whether EAA would predict internalizing symptoms at ages 6-10 in addition to symptoms earlier in childhood. Symptoms at age 2.5 significantly predicted higher levels of symptoms at age $6-10$ (beta $=.125$, SD $=.022, t=5.61, p<.001)$. EAA remained a significant predictor in addition to symptoms at age 2.5 (beta $=.074$, SE $=.027, t=2.77, p=$ .006 ), explaining an additional $6.7 \%$ of variance between individuals, indicating that EAA and early childhood symptoms are at least in part independent predictors of later childhood internalizing symptoms. When symptoms at both age 2.5 and 4 years were considered in a model together with EAA, EAA was associated with later child symptoms at trend level (beta $=.050, \mathrm{SE}=.027, t=1.88, p=.063$ ), explaining an additional $3.1 \%$ of variance between individuals, see Supplementary
Table S7.

In line with the CBCL data, EAA also showed a significant positive association with SDQ-assessed internalizing scores at ages 8-10 after controlling for relevant covariates (beta $=.065, \mathrm{SD}=.026, t=2.46, p=$ .015 ), explaining $4.9 \%$ of variance between individuals. Again no interaction of EAA with time was found (beta $=-.0047, \mathrm{SD}=.029, t$ $=-.162, p=.87$ ).

To visualize the association between EAA at age 6 and internalizing symptoms at ages 6-10, we divided participants into three groups based on EAA scores; EAA $>1$ SD below the mean $(n=23)$, EAA $>1$ SD above the mean $(\mathrm{n}=28)$, and EAA \pm 1 SD around the mean $(\mathrm{n}=96)$. Fig. 2 shows the standardized (log-transformed) CBCL internalizing scores at ages $6-10$ for these 3 EAA groups. CBCL data was standardized to compare symptom scores across time. Fig. 1B furthermore shows a scatterplot of the cross-sectional association between standardized internalizing symptoms at age 6 and EAA at age 6 .

\subsubsection{Externalizing symptoms}

CBCL externalizing symptoms were not significantly different between age 6 and age 10 years (Beta Time $=-.023, \mathrm{SE}=.013, t=-1.741$, $p=.083$ ). Maternal STAI scores significantly positively predicted externalizing symptoms over time (beta $=.0059, \mathrm{SE}=.0026, t=2.241, p$ $=.026$ ), indicating that maternal anxiety was associated with mothers' report on child externalizing symptoms. EAA showed a positive, but non-significant association with externalizing symptoms (beta $=.062, \mathrm{SE}$ $=.033, t=1.882, p=.062$ ), explaining $.8 \%$ of variance between individuals. The interaction of EAA with time was not significant either $(p$ $=.72$ ), see Supplementary Table S8.

In line with the CBCL data, EAA showed a positive, but not statistically significant, association with SDQ-assessed externalizing scores at ages 8-10 (beta $=.0576, \mathrm{SD}=.0296, t=1.950, p=.053$ ), explaining $2.3 \%$ of variance between individuals. The interaction of EAA with time did not reach statistical significance either (beta $=.0446, \mathrm{SD}=.024, t=$ $1.83, p=.069)$.

\section{Discussion}

We found that advanced biological aging at age 6, assessed by EAA, was significantly associated with internalizing symptoms in children from the age of 2.5-10 years. EAA at age 6 was predicted by internalizing symptoms as early as age 2.5 , and was longitudinally associated with internalizing symptoms up to the age of 10 . Furthermore, EAA and early childhood internalizing symptoms were partly independent predictors of later childhood internalizing symptoms, demonstrating the potential of EAA as an independent biological risk index of childhood psychiatric symptoms. Results were confirmed with a second measure of internalizing symptoms at the ages of 8 and 10, and mothers' own anxiety and depression scores did not confound these results.

This is the first study to show associations between epigenetic aging and longitudinally developing psychiatric symptoms in a pediatric sample. Two recent studies, in adolescent girls (Suarez, Lahti, Czamara, Lahti-Pulkkinen, Girchenko et al., 2018) and children aged 8-16 (Sumner, Colich, Uddin, Armstrong, \& McLaughlin, 2019), also report associations between EAA and adolescent mental health, effects that were also specific to internalizing behaviors. Collectively, these findings point to a stronger association between EAA and internalizing symptoms, although we note that in our study EAA was associated with externalizing symptoms at trend level suggesting a similar but weaker effect. Our findings are consistent with other studies that link advanced physical maturation to increased depressive symptoms in childhood (Copeland et al., 2019; Ellis et al., 2019; Lewis et al., 2018). Suarez, Lahti, Czamara, Lahti-Pulkkinen, Knight et al. (2018) furthermore showed that a lower epigenetic gestational age (GA), which is found to predict worse future health outcomes (Arcangeli, Thilaganathan, Hooper, Khan, \& Bhide, 2012), was associated with more child psychiatric problems over time. They calculated epigenetic GA from cord blood 


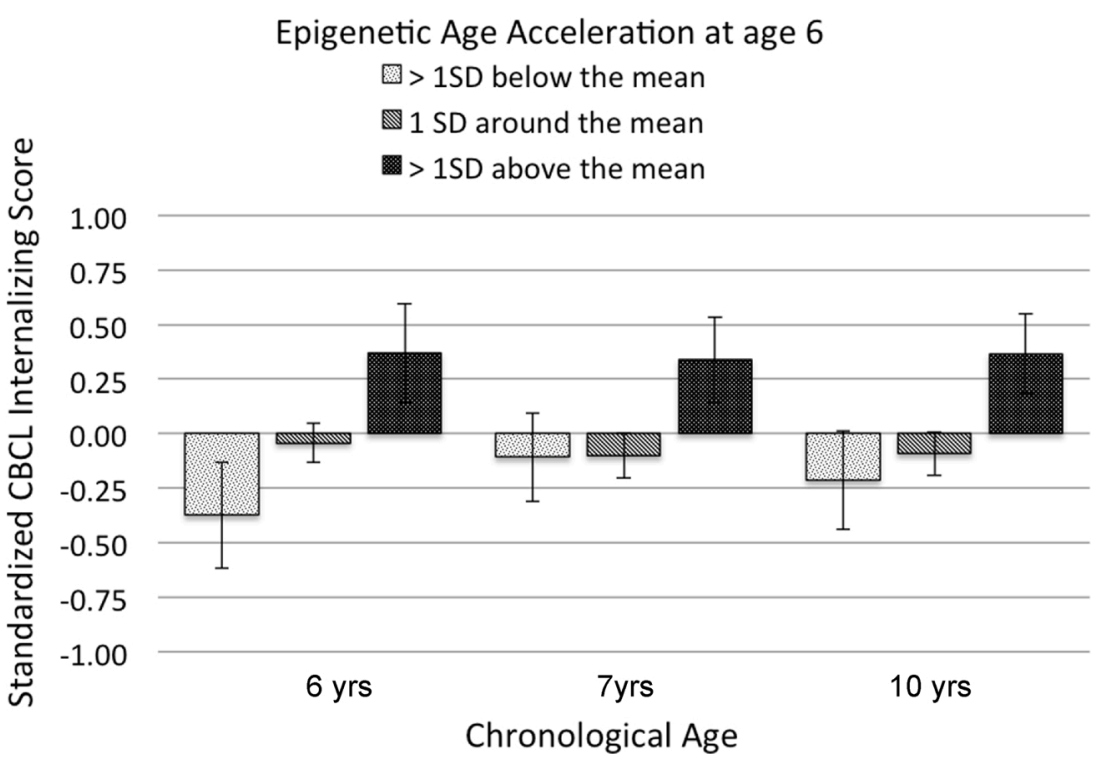

Fig. 2. Associations between Epigenetic Age Acceleration (EAA) at age 6, expressed as distance from the mean (EAA > 1 SD below the mean, EAA $>1$ SD above the mean, and EAA \pm 1SD around the mean), and internalizing symptoms measured with the Child Behavior Checklist (CBCL) over time (6-10 yrs). CBCL values are expressed as standardized scores $(M=0, S D=$ 1 ) around the mean of the chronological age group. Group differences are indicated by $* p<.05,{ }^{* *} p<.01$, yrs $=$ years. with a GA specific epigenetic clock (Knight et al., 2016). Cord blood was not available within the current cohort and as such we cannot determine the relation between epigenetic GA and the Horvath clock, although this would be of interest for future studies.

Future studies should focus on possible underlying mechanisms of the association between EAA and internalizing, and possibly externalizing, symptoms. For example, early life stress is associated with the development of psychiatric symptoms (Blair, Glynn, Sandman, \& Davis, 2011) as well as accelerated epigenetic aging (Simpkin et al., 2016). In our study, internalizing symptoms at age 2.5 predicted EAA at age 6 . It may be that children at risk for internalizing symptoms (due to genetic and/or environmental risk factors) experience adverse life events as more stressful than children without these problems, or experience an increased frequency of adverse life events, which may further increase stress-related effects on epigenetic age acceleration (Zannas et al., 2015). However, as we assessed EAA only at age 6 in the current study, we cannot exclude the possibility that EAA was already evident at age 2.5 or even earlier. Studies including prospective biosampling from birth with repeated measures of both EAA and childhood psychiatric symptoms over time, or examining interventions aimed at reducing psychopathology or EAA, will be important for gaining insight into causality.

The finding that children who exhibit heightened internalizing symptoms also show signs of accelerated biological aging is also of interest given the association between psychiatric disorders and increased risk of aging-related diseases in adulthood (Bennett \& Thomas, 2014; Penninx, 2017) and the growing literature associating EAA with early mortality (Chen et al., 2016; Marioni et al., 2015; Perna et al., 2016). Our findings, if replicated, suggest that the burden of internalizing symptoms, even early in childhood, may influence the pace of aging of biological systems, and raise interesting questions about how to reverse such effects (Horvath \& Raj, 2018). Considering the continuity of problem behavior over time and its association with advanced biological aging, these findings stress the importance of identifying and treating these symptoms and associated risk factors as early in life as possible.

It is important to note that the current study only examined EAA at the age of 6. It is unknown whether EAA at this age is still associated with EAA and internalizing symptoms in adulthood, and whether it is predictive of physical health symptoms. Longitudinal assessment of psychiatric symptoms, health and EAA are needed to disentangle possible underlying mechanisms. This also includes examining the specificity of the psychiatric symptoms that are most strongly associated with EAA, as we found greater evidence for the association of EAA with internalizing than with externalizing symptoms. Also, exploratory analyses at age 6 showed that within the internalizing dimension this association was mostly driven by anxious/depressed and withdrawn behaviors, but not somatic complaints (data not shown). Furthermore, it will be important to integrate other measures of DNA methylation-based or cellular aging (Belsky et al., 2017). For example, shortened leukocyte telomere length at age 38 was associated with the persistence of internalizing disorders from ages 11 to 38 years (Shalev et al., 2014).

Epigenetic age estimates in the current study were significantly lower than participants' chronological age, suggesting a slower pace of epigenetic age in buccal tissue, a feature also observed in neural tissue (Horvath et al., 2015). However, technical features of the $850 \mathrm{~K}$ array and normalization method may also have caused this underestimation of age (McEwen et al., 2018). Future cross-tissue analyses of EAA should determine if buccal tissue is a better proxy for brain-based phenotypes than tissues of a non-ectodermal origin e.g., blood or saliva. While the Horvath clock shows very high accuracy in chronological age prediction (Chen et al., 2016; Horvath, 2013), epigenetic age did not correlate with chronological age in the current sample. This is likely due to the limited age range of the participants during DNA collection, ranging from 5.87 to 6.85 years, but could also be due to the young sample. Previous studies that have used the Horvath-based epigenetic clock in young children also show low correlations with chronological age, but also show significant associations with development-related measures (Simpkin et al., 2016, 2017). Despite the limited correlation with chronological age in young children, epigenetic age acceleration based on the Horvath clock may still reflect underlying biological processes that are informative for child health and development. As such, future research is required to study precursors of epigenetic age acceleration in children, such as trauma and stress during pregnancy or childhood, which could provide a deeper mechanistic understanding of how such environmental stressors become biologically embedded (Aristizabal et al., 2019).

Strengths of this study are the prospective longitudinal design with multiple assessments of psychiatric symptoms during childhood. Nonetheless, the sample was relatively small and thereby under-powered to detect small effect sizes, which may explain the non-significant associations with externalizing symptoms and the genetic predictors of EAA. Similarly, sample size precluded stratified analyses based on sex, which may be important (Suarez, Lahti, Czamara, Lahti-Pulkkinen, Knight et al., 2018). Furthermore, as this is the first report of an association between internalizing symptoms and EAA in childhood it is possible that our relatively large effect sizes represent a 'winner's curse' and highlight the need for replication in independent and larger samples. Also of note 
is that maternal report of childhood symptoms was used, which raises concerns about reporter bias, i.e., anxious mothers may view their children as more anxious. However, adjusting our models for maternal anxiety and depression symptoms at each of the time points that child symptoms were assessed did not change our findings. In an optimal design, objective observer reports or child self-report would be used to discount potential reporter bias effects. Lastly, our sample includes children with low levels of psychiatric symptoms, coming from relatively highly educated Caucasian families from a small area in the Netherlands.

\section{Conclusion}

We report a novel association between child EAA and internalizing symptoms across childhood. Future work should determine the importance of this association for long-term (mental) health outcomes. A next important question is whether addressing internalizing symptoms in children, or the stressful environments they experience, will stop the progress of, or even reduce accelerated epigenetic aging. Interestingly, Brody, Yu, Chen, Beach, and Miller (2016) showed that a family-centered prevention program ameliorated the longitudinal association between risky family processes and epigenetic aging. It will also be interesting to examine whether lifestyle programs, including exercise (Quach et al., 2017), might affect EAA and psychiatric symptoms in at-risk children. As EAA has been shown to predict all-cause mortality (Chen et al., 2016), early indicators and prevention of heightened EAA are an important area of further study.

\section{Declaration of Competing Interest}

The authors declare that they have no known competing financial interests or personal relationships that could have appeared to influence the work reported in this paper.

\section{Acknowledgements}

This research was supported by the Netherlands Organization for Scientific Research VENI grant 016.195.197 (RB), VIDI grant 575-25009 (CdeW), and VICI grant 016.Vici.185.038 (CdeW), a Jacobs Foundation Advanced Research Fellowship (CdeW), and a Sara van Dam Project Grant of the Royal Netherlands Academy of Arts and Sciences (RB). IS was supported by the Mark T. Greenberg Early Career Professorship. KOD, MSK and MJM are CIFAR Fellows in the Child and Brain Development Program. Additional support came from the National Institutes of Health, Eunice Kennedy Shriver National Institute of Child Health and Human Development under Award Number P50HD089922, and the Canada First Research Excellence Fund: Healthy Brains for Healthy Lives.

\section{Appendix A. Supplementary data}

Supplementary material related to this article can be found, in the online version, at doi:https://doi.org/10.1016/j.biopsycho.2021.1080 21.

\section{References}

Achenbach, T. M. (2007). Manual for the ASEBA school-age forms \& profiles an integrated system of multi-informant assessment. Research Center for Children, 82, 60-90.

Achenbach, T. M., \& Rescorla, L. A. (2000). Validity of ASEBA preschool scales. Manual for the ASEBA preschool forms and profiles. Retrieved from http://www.aseba.org/or dering/ASEBAReliability\%26Validity-Pre-school.pdf.

Arcangeli, T., Thilaganathan, B., Hooper, R., Khan, K. S., \& Bhide, A. (2012). Neurodevelopmental delay in small babies at term: A systematic review. Ultrasound in Obstetrics and Gynecology, 40(3), 267-275. https://doi.org/10.1002/uog.11112

Aristizabal, M. J., Anreiter, I., Halldorsdottir, T., Odgers, C. L., McDade, T. W., Goldenberg, A., ... O'Donnell, K. J. (2019). Biological embedding of experience: A primer on epigenetics. Proceedings of the National Academy of Sciences. https://doi. org/10.1073/pnas.1820838116

Beijers, R., Jansen, J., Riksen-Walraven, M., \& de Weerth, C. (2010). Maternal prenatal anxiety and stress predict infant illnesses and health complaints. Pediatrics, 126(2), e401-e409. https://doi.org/10.1542/peds.2009-3226

Belsky, D. W., Moffitt, T. E., Cohen, A. A., Corcoran, D. L., Levine, M. E., Prinz, J. A., ... Caspi, A. (2017). Eleven telomere, epigenetic clock, and biomarker-composite quantifications of biological aging: Do they measure the same thing? American Journal of Epidemiology, 187(6), 1220-1230. https://doi.org/10.1093/aje/kwx346

Bennett, S., \& Thomas, A. J. (2014). Depression and dementia: Cause, consequence or coincidence? Maturitas, 79(2), 184-190. https://doi.org/10.1016/j. maturitas.2014.05.009

Binder, A. M., Corvalan, C., Mericq, V., Pereira, A., Santos, J. L., Horvath, S., . Michels, K. B. (2018). Faster ticking rate of the epigenetic clock is associated with faster pubertal development in girls. Epigenetics, 13(1), 85-94. https://doi.org/ 10.1080/15592294.2017.1414127

Blair, M. M., Glynn, L. M., Sandman, C. A., \& Davis, E. P. (2011). Prenatal maternal anxiety and early childhood temperament. Stress. https://doi.org/10.3109/ 10253890.2011 .594121

Brody, G. H., Yu, T., Chen, E., Beach, S. R. H., \& Miller, G. E. (2016). Family-centered prevention ameliorates the longitudinal association between risky family processes and epigenetic aging. Journal of Child Psychology and Psychiatry and Allied Disciplines, 57(5), 566-574. https://doi.org/10.1111/jcpp.12495

Chen, B. H., Marioni, R. E., Colicino, E., Peters, M. J., Ward-Caviness, C. K., Tsai, P. C., .. Horvath, S. (2016). DNA methylation-based measures of biological age: Metaanalysis predicting time to death. Aging, 8(9), 1844-1865. https://doi.org/ 10.18632/aging. 101020

Copeland, W. E., Worthman, C., Shanahan, L., Costello, E. J., \& Angold, A. (2019). Early pubertal timing and testosterone associated with higher levels of adolescent depression in girls. Journal of the American Academy of Child and Adolescent Psychiatry. https://doi.org/10.1016/j.jaac.2019.02.007

Davis, E. G., Humphreys, K. L., McEwen, L. M., Sacchet, M. D., Camacho, M. C., MacIsaac, J. L., ... Gotlib, I. H. (2017). Accelerated DNA methylation age in adolescent girls: Associations with elevated diurnal cortisol and reduced hippocampal volume. Translational Psychiatry, 7(8), Article e1223. https://doi.org/ 10.1038/tp.2017.188

Ellis, R., Fernandes, A., Simmons, J. G., Mundy, L., Patton, G., Allen, N. B., ... Whittle, S. (2019). Relationships between adrenarcheal hormones, hippocampal volumes and depressive symptoms in children. Psychoneuroendocrinology, 104, 55-63. https://doi. org/10.1016/j.psyneuen.2019.02.016

Fries, G. R., Bauer, I. E., Scaini, G., Wu, M.-J., Kazimi, I. F., Valvassori, S. S., Quevedo, J. (2017). Accelerated epigenetic aging and mitochondrial DNA copy number in bipolar disorder. Translational Psychiatry, 7(12), Article 1283. https://doi. org /10.1038/s41398-017-0048-8

Gassen, N. C., Chrousos, G. P., Binder, E. B., \& Zannas, A. S. (2017). Life stress, glucocorticoid signaling, and the aging epigenome: Implications for aging-related diseases. Neuroscience and Biobehavioral Reviews. https://doi.org/10.1016/j. neubiorev.2016.06.003

Gur, R. E., Moore, T. M., Rosen, A. F. G., Barzilay, R., Roalf, D. R., Calkins, M. E., .. Gur, R. C. (2019). Burden of environmental adversity associated with psychopathology, maturation, and brain behavior parameters in youths. JAMA Psychiatry. https://doi.org/10.1001/jamapsychiatry.2019.0943

Han, L. K. M., Aghajani, M., Clark, S. L., Chan, R. F., Hattab, M. W., Shabalin, A. A., Penninx, B. W. J. H. (2018). Epigenetic aging in major depressive disorder. The American Journal of Psychiatry. https://doi.org/10.1176/appi.ajp.2018.17060595. appi.ajp.2018.1.

Horvath, S. (2013). DNA methylation age of human tissues and cell types. Genome Biology, 14(10), R115. https://doi.org/10.1186/gb-2013-14-10-r115

Horvath, S., \& Raj, K. (2018). DNA methylation-based biomarkers and the epigenetic clock theory of ageing. Nature Reviews Genetics, 19(6), 371-384. https://doi.org/ 10.1038/s41576-018-0004-3

Horvath, S., Mah, V., Lu, A. T., Woo, J. S., Choi, O. W., Jasinska, A. J., ... Coles, L. S. (2015). The cerebellum ages slowly according to the epigenetic clock. Aging. https:// doi.org/10.18632/aging.100742

Kim, S., Wyckoff, J., Morris, A.-T., Succop, A., Avery, A., Duncan, G. E., Jazwinski, S. M. (2018). DNA methylation associated with healthy aging of elderly twins. GeroScience, 40(5-6), 469-484. https://doi.org/10.1007/s11357-018-0040-0

Knight, A. K., Craig, J. M., Theda, C., Bïi $i^{1 / 2 k v a d-H a n s e n, ~ M ., ~ B y b j e r g-G r a u h o l m, ~ J ., ~}$ Hansen, C. S., ... Smith, A. K. (2016). An epigenetic clock for gestational age at birth based on blood methylation data. Genome Biology. https://doi.org/10.1186/s13059016-1068-Z

Lewis, G., Ioannidis, K., van Harmelen, A.-L., Neufeld, S., Stochl, J., Lewis, G., ... Goodyer, I. (2018). The association between pubertal status and depressive symptoms and diagnoses in adolescent females: A population-based cohort study. PloS One, 13(6), Article e0198804. https://doi.org/10.1371/journal.pone.0198804

Lu, A. T., Hannon, E., Levine, M. E., Hao, K., Crimmins, E. M., Lunnon, K., ... Horvath, S. (2016). Genetic variants near MLST8 and DHX57 affect the epigenetic age of the cerebellum. Nature Communications. https://doi.org/10.1038/ncomms10561

Lu, A. T., Xue, L., Salfati, E. L., Chen, B. H., Ferrucci, L., Levy, D., ... Horvath, S. (2018). GWAS of epigenetic aging rates in blood reveals a critical role for TERT. Nature Communications, 9(1), 387. https://doi.org/10.1038/s41467-017-02697-5

Marioni, R. E., Shah, S., McRae, A. F., Chen, B. H., Colicino, E., Harris, S. E. ... Deary, I. J. (2015). DNA methylation age of blood predicts all-cause mortality in later life. Genome Biology, 16(1), 25. https://doi.org/10.1186/s13059-015-0584-6

McEwen, L. M., Jones, M. J., Lin, D. T. S., Edgar, R. D., Husquin, L. T., MacIsaac, J. L., .. Kobor, M. S. (2018). Systematic evaluation of DNA methylation age estimation with 
common preprocessing methods and the Infinium MethylationEPIC BeadChip array. Clinical Epigenetics. https://doi.org/10.1186/s13148-018-0556-2

Mieloo, C. L., Bevaart, F., Donker, M. C. H., van Oort, F. V.a., Raat, H., \& Jansen, W. (2014). Validation of the SDQ in a multi-ethnic population of young children. European Journal of Public Health, 24(1), 26-32. https://doi.org/10.1093/eurpub/ ckt100

Murabito, J. M., Zhao, Q., Larson, M. G., Rong, J., Lin, H., Benjamin, E. J., ... Lunetta, K. L. (2018). Measures of biologic age in a community sample predict mortality and age-related disease: The Framingham offspring study. The Journals of Gerontology: Series A, 73(6), 757-762. https://doi.org/10.1093/gerona/glx144

Penninx, B. W. J. H. (2017). Depression and cardiovascular disease: Epidemiological evidence on their linking mechanisms. Neuroscience \& Biobehavioral Reviews. https:// doi.org/10.1016/j.neubiorev.2016.07.003

Perna, L., Zhang, Y., Mons, U., Holleczek, B., Saum, K. U., \& Brenner, H. (2016). Epigenetic age acceleration predicts cancer, cardiovascular, and all-cause mortality in a German case cohort. Clinical Epigenetics, 8(1). https://doi.org/10.1186/s13148016-0228-Z

Pop, V. J., Komproe, I. H., \& van Son, M. J. (1992). Characteristics of the Edinburgh post natal depression scale in the Netherlands. Journal of Affective Disorders, 26(2), 105-110. https://doi.org/10.1016/0165-0327(92)90041-4

Price, A. L., Patterson, N. J., Plenge, R. M., Weinblatt, M. E., Shadick, N. A., \& Reich, D. (2006). Principal components analysis corrects for stratification in genome-wide association studies. Nature Genetics. https://doi.org/10.1038/ng1847

Quach, A., Levine, M. E., Tanaka, T., Lu, A. T., Chen, B. H., Ferrucci, L., ... Horvath, S. (2017). Epigenetic clock analysis of diet, exercise, education, and lifestyle factors. Aging, 9(2), 419-446. https://doi.org/10.18632/aging.101168

Reef, J., Diamantopoulou, S., van Meurs, I., Verhulst, F., \& van der Ende, J. (2009). Child to adult continuities of psychopathology: A 24-year follow-up. Acta Psychiatrica Scandinavica, 120(3), 230-238. https://doi.org/10.1111/j.1600-0447.2009.01422.x

Shalev, I., Moffitt, T. E., Braithwaite, A. W., Danese, A., Fleming, N. I., GoldmanMellor, S., ... Caspi, A. (2014). Internalizing disorders and leukocyte telomere erosion: A prospective study of depression, generalized anxiety disorder and postTraumatic stress disorder. Molecular Psychiatry, 19(11), 1163-1170. https://doi.org/ 10.1038/mp.2013.183

Simpkin, A. J., Hemani, G., Suderman, M., Gaunt, T. R., Lyttleton, O., Mcardle, W. L., ... Smith, G. D. (2016). Prenatal and early life influences on epigenetic age in children A study of mother-offspring pairs from two cohort studies. Human Molecular Genetics, 25(1), 191-201. https://doi.org/10.1093/hmg/ddv456

Simpkin, A. J., Howe, L. D., Tilling, K., Gaunt, T. R., Lyttleton, O., McArdle, W. L., ... Relton, C. L. (2017). The epigenetic clock and physical development during childhood and adolescence: Longitudinal analysis from a UK birth cohort.
International Journal of Epidemiology, 46(2), Article dyw307. https://doi.org/ 10.1093/ije/dyw307

Smith, A. K., Kilaru, V., Klengel, T., Mercer, K. B., Bradley, B., Conneely, K. N., ... Binder, E. B. (2015). DNA extracted from saliva for methylation studies of psychiatric traits: Evidence tissue specificity and relatedness to brain. American Journal of Medical Genetics, Part B: Neuropsychiatric Genetics. https://doi.org/ 10.1002/ajmg.b.32278

Spielberger, C. D. (1983). State-trait anxiety inventory (STAI). Mind Garden, 94061(650), 261-3500.

Suarez, A., Lahti, J., Czamara, D., Lahti-Pulkkinen, M., Girchenko, P., Andersson, S., ... Raikkonen, K. (2018). The epigenetic clock and pubertal, neuroendocrine, psychiatric, and cognitive outcomes in adolescents. Clinical Epigenetics, 10(1), 96. https://doi.org/10.1186/s13148-018-0528-6

Suarez, A., Lahti, J., Czamara, D., Lahti-Pulkkinen, M., Knight, A. K., Girchenko, P., ... Räikkönen, K. (2018). The epigenetic clock at birth: Associations with maternal antenatal depression and child psychiatric problems. Journal of the American Academy of Child \& Adolescent Psychiatry, O(0). https://doi.org/10.1016/j jaac.2018.02.011

Sumner, J. A., Colich, N. L., Uddin, M., Armstrong, D., \& McLaughlin, K. A. (2019). Early experiences of threat, but not deprivation, are associated with accelerated biological aging in children and adolescents. Biological Psychiatry. https://doi.org/10.1016/j. biopsych.2018.09.008

Tollenaar, M. S., Beijers, R., Jansen, J., Riksen-Walraven, J. M. A., \& De Weerth, C. (2011). Maternal prenatal stress and cortisol reactivity to stressors in human infants. Stress, 14(1). https://doi.org/10.3109/10253890.2010.499485

Whiteford, H. A., Degenhardt, L., Rehm, J., Baxter, A. J., Ferrari, A. J., Erskine, H. E., .. Vos, T. (2013). Global burden of disease attributable to mental and substance use disorders: Findings from the Global Burden of Disease Study 2010. The Lancet. https://doi.org/10.1016/S0140-6736(13)61611-6

Wolf, E. J., \& Schnurr, P. P. (2016). PTSD-related cardiovascular disease and accelerated cellular aging. Psychiatric Annals, 46, 527-532. Retrieved from http://www.ncbi. nlm.nih.gov/pubmed/27990033.

Zannas, A. S., Arloth, J., Carrillo-Roa, T., Iurato, S., Röh, S., Ressler, K. J., .. Mehta, D. (2015). Lifetime stress accelerates epigenetic aging in an urban, African American cohort: Relevance of glucocorticoid signaling. Genome Biology, 16(1), 1-12. https:// doi.org/10.1186/s13059-015-0828-5

Zheng, S. C., Webster, A. P., Dong, D., Feber, A., Graham, D. G., Sullivan, R., .. Teschendorff, A. E. (2018). A novel cell-type deconvolution algorithm reveals substantial contamination by immune cells in saliva, buccal and cervix. Epigenomics, 10(7), 925-940. https://doi.org/10.2217/epi-2018-0037 